\title{
BMJ Open External validation of the quick Sequential Organ Failure Assessment score for mortality and bacteraemia risk evaluation in Japanese patients undergoing haemodialysis: a retrospective multicentre cohort study
}

Hiroki Nishiwaki, ${ }^{1,2,3}$ Sho Sasaki, ${ }^{2,4}$ Takeshi Hasegawa, ${ }^{1,2,3}$ Fumihiko Sasai, ${ }^{1}$ Hiroo Kawarazaki, ${ }^{5}$ Shun Minatoguchi, ${ }^{6,7}$ Daisuke Uchida, ${ }^{8}$ Kenichiro Koitabashi, ${ }^{9}$ Takaya Ozeki, ${ }^{7,10}$ Fumihiko Koiwa, ${ }^{1}$ the JOINT-KD collaborators

To cite: Nishiwaki $\mathrm{H}$, Sasaki S, Hasegawa T, et al. External validation of the quick Sequential Organ Failure Assessment score for mortality and bacteraemia risk evaluation in Japanese patients undergoing haemodialysis: a retrospective multicentre cohort study. BMJ Open 2019;9:e028856. doi:10.1136/ bmjopen-2018-028856

- Prepublication history for this paper is available online. To view please visit the journal online (http://dx.doi.org/10. 1136/bmjopen-2018-028856).

Received 2 January 2019 Revised 9 April 2019 Accepted 17 May 2019
Check for updates

(c) Author(s) (or their employer(s)) 2019. Re-use permitted under CC BY-NC. No commercial re-use. See rights and permissions. Published by BMJ.

For numbered affiliations see end of article.

Correspondence to

Dr Takeshi Hasegawa;

tahasegawa-npr@umin.net

\section{ABSTRACT}

Objectives We aimed to examine the validity of the quick Sequential Organ Failure Assessment (qSOFA) score for mortality and bacteraemia risk assessment in Japanese haemodialysis patients.

Design This is a retrospective multicentre cohort study. Setting The six participating hospitals are tertiary-care institutions that receive patients on an emergency basis and provide primary, secondary and tertiary care. The other participating hospital is a secondary-care institution that receives patients on an emergency basis and provides both primary and secondary care.

Participants This study included haemodialysis outpatients admitted for bacteraemia suspicion, who had blood drawn for cultures within 48 hours of their initial admission.

Primary and secondary outcome measures The primary outcome measure was overall in-hospital mortality. Secondary outcomes included 28-day in-hospital mortality and the incidence of bacteraemia diagnosed based on blood culture findings. The discrimination, calibration and test performance of the QSOFA score were assessed. Missing data were handled using multiple imputation.

Results Among the 507 haemodialysis patients admitted with bacteraemia suspicion between August 2011 and July 2013 , the overall in-hospital mortality was $14.6 \%$ (74/507), the 28-day in-hospital mortality was $11.1 \%$ (56/507) and the incidence of bacteraemia, defined as a positive blood culture, was $13.4 \%$ (68/507). For predicting in-hospital mortality among haemodialysis patients, the area under the receiver operating characteristic curve was $0.61(95 \% \mathrm{Cl} 0.56-0.67)$ for a qSOFA score $\geq 2$. The Hosmer-Lemeshow $\chi^{2}$ statistics for the qSOFA score as a predictor of overall and 28-day in-hospital mortality were $5.72(p=0.02)$ and $7.40(p<0.01)$, respectively. Conclusion On external validation, the qSOFA score exhibited low diagnostic accuracy and miscalibration for in-hospital mortality and bacteraemia among haemodialysis patients.
Strengths and limitations of this study

This is the first study to assess the diagnostic performance of the quick Sequential Organ Failure Assessment (qSOFA) score for in-hospital mortality and bacteraemia among haemodialysis patients, according to the Transparent Reporting of a multivariable prediction model for Individual Prognosis or Diagnosis statement.

- We could not precisely determine the performance of the qSOFA score in haemodialysis patients with symptoms that did not warrant blood culture evaluation because we did not evaluate the reasons blood was drawn for culture.

- We used consecutive data of haemodialysis patients suspected of having bacteraemia, which is expected to increase the generalisability of our findings.

- Our cohort contains patients who used antibiotics during the week leading up to the hospital visit, which could have decreased infection-related mortality and decreased the rate of positive blood cultures.

- Our cohort was geographically and temporally different from the cohort used to derive the qSOFA criteria, which enabled us to perform a true external validation study.

\section{INTRODUCTION}

Patients undergoing haemodialysis are at high risk for bloodstream infections due to immunocompromised status and daily punctures required for vascular access. ${ }^{1}$ Moreover, the morbidity and mortality of bacteraemia are higher among haemodialysis patients than in the general population, ${ }^{2-10}$ as is the incidence of Staphylococcus aureus bloodstream infections. ${ }^{11}$ Therefore, appropriate 
diagnosis and timely treatment of bacteraemia are of critical importance in haemodialysis patients.

While many risk stratification tools are available for the general population, their diagnostic accuracy is likely to differ when applied in specific populations. Adequate validation of population-specific diagnostic performance is particularly important in high-risk populations such as haemodialysis patients. For example, we previously reported that the Systemic Inflammatory Response Syndrome (SIRS) score has low sensitivity for predicting bloodstream infections in haemodialysis patients (SIRS score $\geq 2$ : sensitivity, $71.9 \%$; specificity, $45.2 \%$; positive likelihood ratio, 1.31; negative likelihood ratio, 0.62). ${ }^{12}$ These previous findings suggested that the prediction criteria for bacteraemia or sepsis, which are well-established for the general population, might have different diagnostic accuracy among haemodialysis patients.

We also proposed a clinical prediction rule for bacteraemia among haemodialysis outpatients with suspicion of bacteraemia (BAC-HD). ${ }^{13}$ The BAC-HD score takes into account body temperature, heart rate, C-reactive protein levels, alkaline phosphatase levels and use of antibiotics within the week leading up to the assessment. A BAC-HD score $\geq 2$ was useful for predicting bacteraemia in haemodialysis patients (sensitivity, 89.6\%; specificity, 51.4\%; positive likelihood ratio, 1.8; negative likelihood ratio, 0.2 ; area under the curve (AUC), 0.76). ${ }^{13}$

The quick Sequential Organ Failure Assessment (qSOFA) score was introduced as a novel risk-stratification tool intended for use outside the intensive care unit (ICU). The qSOFA score is based on three clinical criteria: systolic hypotension, defined as a systolic blood pressure $\leq 100 \mathrm{~mm} \mathrm{Hg}$; tachypnea, defined as a respiratory rate $\geq 22$ breaths $/ \mathrm{min}$; and altered mentation. ${ }^{14}$ In a previous study, the qSOFA score showed predictive validity (area under the receiver operating characteristic curve, $0.81 ; 95 \%$ CI 0.80 to 0.82 ) for sepsis in non-ICU patients with suspected infection identified as the combination of antibiotics use and body fluid cultures. ${ }^{14}$ Several studies have been conducted to validate the diagnostic performance of the qSOFA score among patients in various settings or with specific comorbidities. ${ }^{15-21}$ However, the validity of qSOFA for risk evaluation in haemodialysis patients has not been confirmed to date.

In the present study, we aimed to examine the external validity of qSOFA as an easy-to-use tool for rapid evaluation of the risk of in-hospital death and bacteraemia in patients undergoing haemodialysis.

\section{MATERIALS AND METHODS}

\section{Study design and participants}

Seven hospitals participated in this multicentre, retrospective cohort study of maintenance haemodialysis patients. The six participating hospitals are tertiary-care institutions that receive patients on an emergency basis and provide primary, secondary and tertiary care. The other participating hospital is a secondary-care institution that receives patients on an emergency basis and provides both primary and secondary care. The study results are reported in accordance with the Transparent Reporting of a multivariable prediction model for Individual Prognosis Or Diagnosis (TRIPOD) statement. ${ }^{22}$

The present study included consecutive haemodialysis patients with suspected bacteraemia who visited the outpatient department or emergency room between August 2011 and July 2013 and had blood drawn for cultures within 48 hours of their initial arrival at the hospital. The exclusion criteria of this study were as follows: age below 18 years; low frequency of haemodialysis (less than once per week); combination dialysis regimen (peritoneal dialysis and haemodialysis); admission within $\leq 2$ weeks of haemodialysis initiation; and referral from another hospital.

\section{Outcome measures}

The primary outcome measure was the overall in-hospital mortality. Considering the findings of previous validation studies, 28-day in-hospital mortality was defined as the secondary outcome. Bacteraemia incidence was another secondary outcome measure in this study. Bacteraemia was diagnosed based on the results of blood cultures at the time of the patient's visit. Specifically, the diagnosis of bacteraemia was made if the blood cultures were positive for any bacteria and there was no suspicion of contamination. Contamination was considered the most probable cause of positive blood culture results if only one of two sets of culture bottles was positive, or if all detected bacterial species were known to be common contaminants (ie, diphtheroids, Bacillus sp, Propionibacterium sp, micrococci, Corynebacterium sp, and coagulase-negative staphylococci). Finally, an external consensus panel of two physicians well trained in infectious diseases determined whether a culture was contaminated or not, based on the above definitions and their clinical expertise.

\section{Method of measurement}

The following data were extracted from the medical records: age; sex; dialysis vintage; cause of end-stage renal disease; vital signs at the time of the first visit, including body temperature, systolic blood pressure, pulse rate, respiratory rate, percutaneous oxygen saturation, Glasgow Coma Scale (GCS) score, and Japan Coma Scale (JCS) score $^{2324}$; comorbidities; type of vascular access; history of bacteraemia; medication use including antibiotics use within the week leading up to the hospital visit; and laboratory data at the time of the hospital visit, including white blood cell count, platelet count, serum albumin levels and C-reactive protein levels.

A positive qSOFA result ( $q$ SOFA score $\geq 2$ ) was defined in patients who fulfilled two or more of the following criteria at the same time: systolic blood pressure $\leq 100 \mathrm{~mm} \mathrm{Hg}$, respiratory rate $\geq 22$ breaths $/ \mathrm{min}$ and altered mentation. The qSOFA score ranges from 0 to 3 , with each criterion being worth one point. The initial qSOFA scores were established according to the patients' vital signs and 
mental status within 24 hours of arrival. Altered mentation was defined as a GCS score $<13$. If the JCS score was reported instead of the GCS score, the following equivalence was applied: a JCS score of 0 (alert) was considered to correspond to a GCS score of 15 , while a JCS score of 300 (no motor response) was considered to correspond to a GCS score of $3{ }^{23}{ }^{24}$ Converting JCS scores to GCS scores has not been validated. Thus, the other value of the JCS score was considered as missing data and handled using multiple imputation.

\section{Statistical analysis}

Data are presented as median values and IQRs for continuous variables, and as frequencies and percentages for categorical variables. The number of patients who had complete data for each qSOFA category is listed.

In the analysis of the discrimination, calibration and performance of the qSOFA, primary imputation was employed to handle missing values for covariates, assuming that data were missing at random. To impute the missing values, we constructed multiple regression models including variables that could potentially explain the missing data, as well as variables correlated with the outcome. The results obtained across 100 imputed data sets were combined by averaging, and SE were adjusted to reflect both within-imputation and between-imputation variability. These estimates and their $\mathrm{SE}$ were combined using Rubin's rules.

For each qSOFA score cut-off $(\geq 1, \geq 2$, and 3$)$, the discrimination for predicting overall in-hospital mortality, 28-day in-hospital mortality and bacteraemia was assessed as the AUC considering data for all patients. The calibration of the risk score predictions was assessed by plotting observed proportions versus predicted probabilities and by calculating the Hosmer-Lemeshow $\chi^{2}$ statistic. Performance was evaluated as sensitivity, specificity, positive and negative likelihood ratio, and positive and negative predictive value.

The minimum required sample size was estimated at 500 patients, based on the TRIPOD statement. ${ }^{22}$ All analyses were performed using the statistical software programmes Stata V.14.2 (StataCorp) and R V.3.4.1 (The R Foundation for Statistical Computing, https://www.rproject.org). Two-sided significance was set at 0.05 .

\section{Patient and public involvement}

The public and patients were not involved in the development of the research question and outcome measures, study design or study recruitment. We will disseminate the final results to the study participants after they are published in a peer-reviewed journal.

\section{RESULTS}

A total of 507 haemodialysis patients treated during the study period fulfilled the criteria for inclusion in this study. The overall in-hospital mortality in this population was $14.6 \%$ (74/507), whereas 28-day in-hospital mortality
Table 1 Final diagnoses and corresponding mortality rates

Mortality, $\mathbf{n}$

\begin{tabular}{lcc} 
Final diagnosis & Patients (n) & (\%) \\
\hline $\begin{array}{l}\text { System with Infection } \\
\text { Heart and vessels }\end{array}$ & 11 & $5(45.5)$ \\
\hline $\begin{array}{l}\text { Musculoskeletal system } \\
\text { Infectious disease related to } \\
\text { the vascular access }\end{array}$ & 18 & $5(27.8)$ \\
Intra-abdominal & 24 & $6(25.0)$ \\
Respiratory system & 54 & $12(22.2)$ \\
Urinary organ & 99 & $9(9.1)$ \\
Skin & 32 & $2(6.3)$ \\
Other & 24 & $1(4.2)$ \\
$\quad$ Unknown & 38 & $7(18.4)$ \\
Non-infectious disease & 150 & $5(8.8)$ \\
\hline
\end{tabular}

was $11.1 \%(56 / 507)$ and incidence of positive blood culture was $13.4 \%$ (68/507). In-hospital mortality rates were $5.2 \%$ among patients with a qSOFA score $<2$ and $29.6 \%$ among those with a qSOFA score $\geq 2$. The corresponding mortality rates among patients with a positive blood culture were $3.9 \%$ and $35.3 \%$, respectively. Table 1 provides a summary of the final diagnoses and corresponding mortality rates.

Of the 507 participants (median age, 73 years), 36.5\% were women. The most common cause of chronic kidney disease was diabetic nephropathy (40.0\%), while the most frequent route of vascular access was arteriovenous fistula $(74.0 \%)$. The mean haemodialysis vintage was 61 months, and $16.4 \%$ of patients had taken antibiotics within the week leading up to the hospital visit (table 2).

The most frequent pathogen in blood cultures was $S$. aureus, accounting for 28 cases of all bacteraemia cases (15 cases involving methicillin-sensitive $S$. aureus infection and 13 cases involving methicillin-resistant $S$. aureus infection). Klebsiella pneumoniae and Escherichia coli were the causal agent in 11 and 9 cases, respectively. Among the 68 patients with bacteraemia, 5 had polymicrobial infection (table 3).

Of the 255 patients with complete data, 140 (54.9\%), $91(35.7 \%), 21(8.2 \%)$ and $3(1.2 \%)$ had qSOFA scores of $0,1,2$ and 3 on hospital arrival. Among the patients with a qSOFA score of 1, tachypnea (respiratory rate $\geq 22$ breaths $/ \mathrm{min}$ ) was the clinical criterion most commonly fulfilled $(61.5 \% ; 56 / 91)$. Among the patients with a qSOFA score of 2 , the combination of altered mentation and tachypnea was the most common $(47.6 \%$; $10 / 21)$.

For predicting in-hospital mortality in haemodialysis patients, the areas under the receiver operating characteristic curves were 0.59 (95\% CI 0.53 to 0.66$)$ for a qSOFA score $\geq 1,0.61$ (95\% CI 0.56 to 0.67$)$ for a score $\geq 2$ and 0.51 (95\% CI 0.49 to 0.53 ) for a score $\geq 3$ (table 4 ). A summary of sensitivity, specificity, positive and negative 
Table 2 Characteristics of haemodialysis outpatients admitted for suspected bacteraemia $(n=507)$



${ }^{*}$ Continuous data are summarised as median (IQR), while categorical data are summarised as frequency and percentage.

†Missing data are summarised as frequency and percentage.

$\ddagger$ Systolic hypotension was defined as systolic BP $\leq 100 \mathrm{~mm} \mathrm{Hg}$.

$\S$ Tachypnea was defined as a respiratory rate of $\geq 22$ breaths $/ \mathrm{min}$.

$\mathrm{AV}$, arteriovenous; BP, blood pressure; ESRD, end-stage renal disease; GCS, Glasgow Coma Scale

likelihood ratios, and positive and negative predictive values for each qSOFA score cut-off is provided in table 4.

The Hosmer-Lemeshow $\chi^{2}$ statistics for the qSOFA score as a predictor of overall in-hospital mortality and

\begin{tabular}{lc}
$\begin{array}{l}\text { Table } 3 \\
\text { patients }\end{array}$ & Pathogens causing bacteraemia in haemodialysis \\
\hline Bacterium & 28 \\
\hline Staphylococcus aureus & 15 \\
Methicillin-sensitive S. aureus & 13 \\
Methicillin-resistant S. aureus & 11 \\
Klebsiella pneumoniae & 9 \\
Escherichia coli & 5 \\
Coagulase-negative Staphylococcus species & 3 \\
Enterococcus faecalis & 2 \\
Clostridium perfringens & 2 \\
Bacteroides species & 2 \\
Enterococcus faecium & 14 \\
Other &
\end{tabular}

28-day in-hospital mortality were $5.72(\mathrm{p}=0.02)$ and 7.40 $(\mathrm{p}<0.01)$, respectively. The observed and predicted overall in-hospital mortality and 28-day in-hospital mortality were compared on calibration plots (figure 1). As the number of patients with a qSOFA score of 3 was too small, calibration analysis considered patients with a qSOFA score of 2 or 3 together (figure 1).

\section{DISCUSSION}

In this study, we investigated the diagnostic accuracy of qSOFA for predicting in-hospital mortality and bacteraemia incidence in haemodialysis patients who presented to the hospital with suspicion of bacteraemia. Overall, the qSOFA criteria had low accuracy for predicting mortality and bacteraemia incidence among such haemodialysis patients.

qSOFA has several advantages including easy bedside application, reliance on very few variables and no requirement for laboratory tests. However, of the recent studies on the validity of qSOFA in the emergency department setting, ${ }^{15-20}$ one reported poor sensitivity 


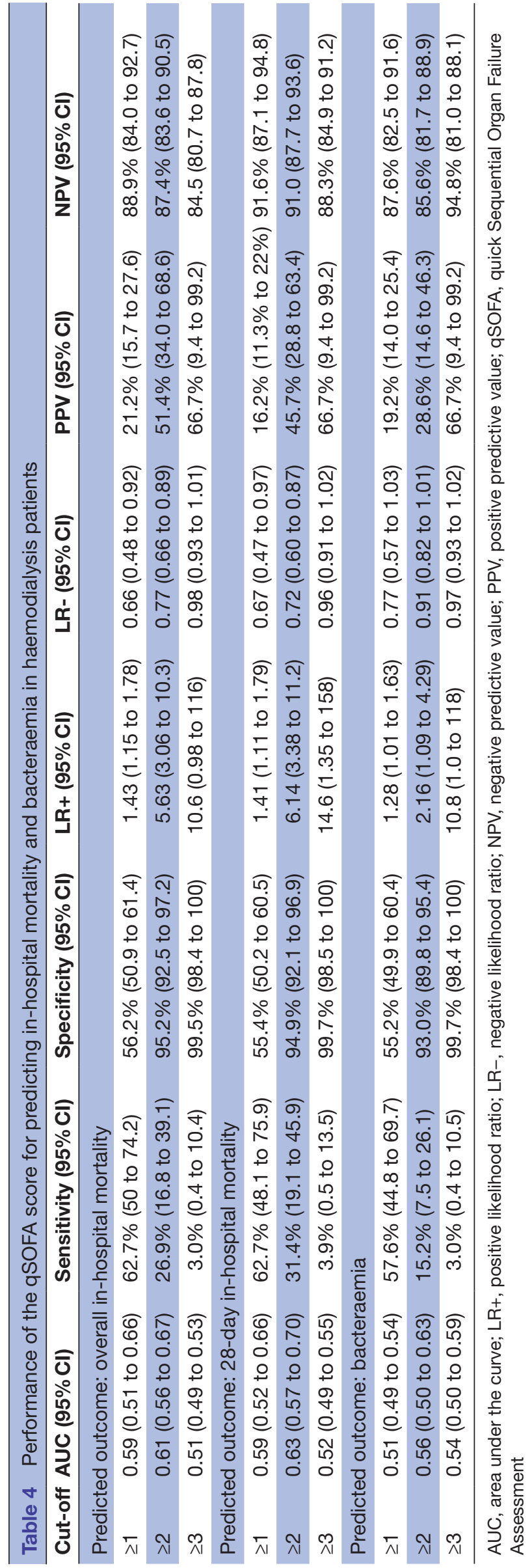

for qSOFA-based out-of-hospital identification of severe sepsis and septic shock. ${ }^{21}$ To the best of our knowledge, the present study represents the first investigation of the external validity of qSOFA for risk stratification of haemodialysis patients with suspicion of infection. Our results revealed that qSOFA exhibits low sensitivity and miscalibration for in-hospital mortality and bacteraemia in haemodialysis patients. In particular, the calibration plot revealed that a qSOFA score of 1 overestimated, while qSOFA score of 2 or 3 underestimated both overall and 28-day in-hospital mortality. There may be several reasons for such findings. First, infection with different causal pathogens may have different manifestations. We confirmed previous observations that $S$. aureus is the most common bacterial pathogen causing bloodstream infection among haemodialysis patients. ${ }^{11}$ Nevertheless, sepsis may have a different causal agent in haemodialysis patients than in the general population; the qSOFA score may not be able to fully account for different clinical presentations. Second, dialysis patients often present with immune system dysfunction and uraemia, as well as with comorbidities such as diabetes mellitus and connective tissue disorder, ${ }^{25}$ which may also affect clinical manifestation, further distinguishing haemodialysis patients from the general population and detrimentally affecting the performance of the qSOFA score. In addition, most dialysis patients have hypertension, ${ }^{26}$ and thus the incidence of hypotension, which is a key qSOFA criterion, may be low in haemodialysis patients with bacteraemia. Third, our baseline data were collected at the time of the hospital visit. One study revealed that a positive qSOFA result (qSOFA score $\geq 2$ ) at hospital presentation and at 3,6 and 24 hours after admission had poor sensitivity and specificity for predicting 28-day mortality. ${ }^{20}$ In other words, the timing of data collection may also affect the performance of the qSOFA score, especially in haemodialysis patients.

Our study has several strong points. First, we included a multicentre cohort of haemodialysis patients, which reduced selection bias. Second, we used multiple imputation, which allowed us to investigate the entire cohort without having to exclude subjects with a relatively mild clinical presentation and thus without a detailed history or laboratory test findings, which would have induced information bias. Third, our cohort was geographically and temporally different from the cohort used to derive the qSOFA criteria, which enabled us to perform a true external validation study.

Several limitations of the present study warrant mention. First, given that we did not evaluate the reasons blood was drawn for culture, we cannot precisely determine the performance of qSOFA in haemodialysis patients with symptoms (eg, fever) that did not warrant blood culture evaluation. However, because it is not possible to predict clinical judgement in such situations, we believe this lack of consideration actually increases the generalisability of our findings, as is the case with the study that developed the clinical prediction rule for bacteraemia in 


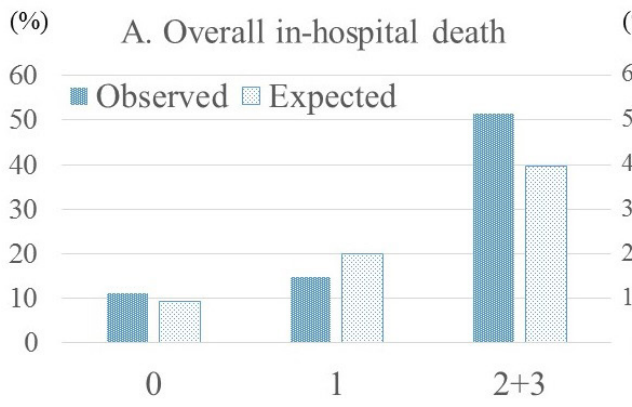

qSOFA score
(\%)

60

50

40

30

20

10

0
B. In-hospital death within 28-day

Observed $\square$ Expected

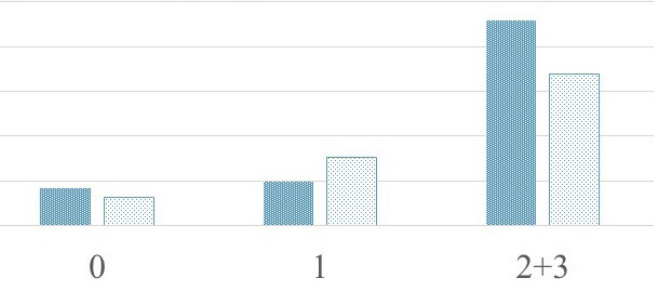

qSOFA score

Figure 1 Observed and predicted in-hospital mortality among haemodialysis outpatients admitted for suspected bacteraemia. (A) Overall in-hospital mortality. (B) 28-day in-hospital mortality. qSOFA, quick Sequential Organ Failure Assessment.

the general population. ${ }^{27}$ Second, our cohort contains patients who used antibiotics during the week leading up to the hospital visit, which could have affected their vital signs at presentation and decreased infection-related mortality and the rate of positive blood cultures. Third, we could not exclude the possibility that some patients had bacteraemia that was not detected on blood culture examination (ie, blood culture-negative bacteraemia), which is considered a limitation of blood culture. Finally, the exact time from hospital arrival to vital sign collection varied, which may have affected the qSOFA score and its relationship with patient prognosis. Employing routinely collected vital signs (eg, vital signs collected at the dialysis centre) for qSOFA score calculation might have provided a better reflection of bacteraemia status; however, vital sign data from the dialysis centres were not available to us at the time of the study.

To summarise, our validation study revealed that, in haemodialysis patients, the qSOFA score exhibits low diagnostic accuracy and miscalibration for in-hospital mortality and bacteraemia. A new prediction score is needed for mortality risk stratification of haemodialysis patients. For bacteraemia risk stratification, the BAC-HD score may outperform the qSOFA score in terms of predicting value.

\section{Author affiliations}

${ }^{1}$ Division of Nephrology, Department of Medicine, Showa University Fujigaoka

Hospital, Yokohama, Japan

${ }^{2}$ Center for Innovative Research for Communities and Clinical Excellence,

Fukushima Medical University, Fukushima, Japan

${ }^{3}$ Research Administration Center (SURAC), Showa University, Tokyo, Japan

${ }^{4}$ Department of Nephrology/Clinical Research Support Office, lizuka Hospital, lizuka, Japan

${ }^{5}$ Department of Nephrology, Inagi Municipal Hospital, Inagi, Japan

${ }^{6}$ Department of Nephrology, Chubu Rosai Hospital, Nagoya, Japan

${ }^{7}$ Department of Nephrology, Nagoya University Graduate School of Medicine,

Nagoya, Japan

${ }^{8}$ Department of Nephrology and Hypertension, Kawasaki Municipal Tama Hospital, Kawasaki, Japan

${ }^{9}$ Division of Nephrology and Hypertension, Saint Marianna University School of Medicine, Kawasaki, Japan

${ }^{10}$ Department of Nephrology, Toyohashi Municipal Hospital, Toyohashi, Japan

Acknowledgements We thank the JOINT-KD collaborators and Naomi Ako (Division of Nephrology and Hypertension, Department of Internal Medicine, St.
Marianna University School of Medicine, Kawasaki) for their intellectual support in the management of this study.

Collaborators The JOINT-KD collaborators included Masahide Furusho (Department of Nephrology, Kagoshima Medical Center, Kagoshima, Japan), Atsushi Nomura (Department of Immunology, Juntendo University School of Medicine, Tokyo, Japan), Hideaki Shimizu (Department of Nephrology, Daido Hospital, Nagoya, Japan), Akihiro Ryuge (Department of Nephrology, Nagoya University Graduate School of Medicine, Nagoya, Japan), Takahiro Imaizumi (Department of Nephrology, Nagoya University Graduate School of Medicine, Nagoya, Japan), Masaru Murasawa (Department of Nephrology, St. Marianna University School of Medicine Yokohama City Seibu Hospital, Yokohama, Japan), Yoshihiko Raita (Department of Nephrology, Okinawa Chubu Hospital, Okinawa, Japan), Yosuke Saka (Department of Nephrology, Kasugai Municipal Hospital, Kasugai, Japan), Masahiko Yazawa (Division of Nephrology and Hypertension, Department of Internal Medicine, St. Marianna University School of Medicine, Kawasaki, Japan), Maho Terashita (Division of Nephrology and Hypertension, Department of Internal Medicine, St. Marianna University School of Medicine, Kawasaki, Japan), Yasunori Suzuki (Division of Nephrology and Rheumatology, Ishikawa Prefectural Central Hospital, Kanazawa, Japan), Yoshiro Fujita (Department of Nephrology, Chubu Rosai Hospital, Nagoya, Japan), Minoru Murakami (Department of Nephrology, Saku Central Hospital , Saku, Japan), Yuichi Maruta (Division of Nephrology, Department of Medicine, Showa University Fujigaoka Hospital, Yokohama, Japan), Megumi Oikawa (Division of Nephrology, Department of Medicine, Showa University Fujigaoka Hospital, Yokohama, Japan), Hisako Saito (Division of Nephrology and Endocrinology, The University of Tokyo, Tokyo, Japan), Aya Mizukami(Division of Nephrology, Department of Medicine, Showa University Fujigaoka Hospital, Yokohama, Japan), Shinya Omiya (Division of Nephrology, Department of Medicine, Showa University Fujigaoka Hospital, Yokohama, Japan) and Yugo Shibagaki (Division of Nephrology and Hypertension, Department of Internal Medicine, St. Marianna University School of Medicine, Kawasaki).

Contributors All authors have seen and approved the final version of the manuscript for publication. HN was responsible for the research idea and study design. HN, SS, FS, HK, SM, DU, KK, TO and JOINT-KD collaborators were responsible for data acquisition. HN, SS and TH were responsible for data analysis/ interpretation. HN and SS were responsible for statistical analysis. TH and FK provided supervision or mentorship. Each author contributed important intellectual content during manuscript drafting or revision and accepts accountability for the overall work by ensuring that questions pertaining to the accuracy or integrity of any portion of the work are appropriately investigated and resolved.

Funding The authors have not declared a specific grant for this research from any funding agency in the public, commercial or not-for-profit sectors.

Competing interests None declared.

Patient consent for publication Not required.

Ethics approval The present study was approved by the ethics committees of St. Marianna Medical University (No. 2713) and Showa University Fujigaoka Hospital (No. 2014072) and was conducted in accordance with the ethical standards set out in the Declaration of Helsinki and its later amendments.

Provenance and peer review Not commissioned; externally peer reviewed.

Data sharing statement You can use the anonymous data set used in this study, only if the reason for reusing the data is clear and it is determined that the reason 
is appropriate. We will keep the data for the data retention period applied for our Institutional Review Boards.

Open access This is an open access article distributed in accordance with the Creative Commons Attribution Non Commercial (CC BY-NC 4.0) license, which permits others to distribute, remix, adapt, build upon this work non-commercially, and license their derivative works on different terms, provided the original work is properly cited, appropriate credit is given, any changes made indicated, and the use is non-commercial. See: http://creativecommons.org/licenses/by-nc/4.0/.

\section{REFERENCES}

1. Laupland KB, Gregson DB, Zygun DA, et al. Severe bloodstream infections: a population-based assessment. Crit Care Med 2004;32:992-7.

2. Hoen B, Paul-Dauphin A, Hestin D, et al. EPIBACDIAL: a multicenter prospective study of risk factors for bacteremia in chronic hemodialysis patients. J Am Soc Nephrol 1998;9:869-76.

3. Dopirak M, Hill C, Oleksiw M, et al. Surveillance of hemodialysisassociated primary bloodstream infections: the experience of ten hospital-based centers. Infect Control Hosp Epidemiol 2002;23:721-4.

4. Gilad J, Eskira S, Schlaeffer F, et al. Surveillance of chronic haemodialysis-associated infections in southern Israel. Clin Microbiol Infect 2005; 11:547-52.

5. Ishani A, Collins AJ, Herzog CA, et al. Septicemia, access and cardiovascular disease in dialysis patients: the USRDS Wave 2 study. Kidney Int 2005;68:311-8.

6. Chan KE, Warren HS, Thadhani RI, et al. Prevalence and outcomes of antimicrobial treatment for Staphylococcus aureus bacteremia in outpatients with ESRD. J Am Soc Nephrol 2012;23:1551-9.

7. Fysaraki M, Samonis G, Valachis A, et al. Incidence, clinical, microbiological features and outcome of bloodstream infections in patients undergoing hemodialysis. Int J Med Sci 2013;10:1632-8.

8. Eleftheriadis T, Liakopoulos V, Leivaditis K, et al. Infections in hemodialysis: a concise review - Part 1: bacteremia and respiratory infections. Hippokratia 2011;15:12-17.

9. Sarnak MJ, Jaber BL. Mortality caused by sepsis in patients with end-stage renal disease compared with the general population. Kidney Int 2000;58:1758-64.

10. Foley RN, Guo H, Snyder JJ, et al. Septicemia in the United States dialysis population, 1991 to 1999. J Am Soc Nephrol 2004;15:1038-45.

11. Nielsen LH, Jensen-Fangel S, Benfield T, et al. Risk and prognosis of Staphylococcus aureus bacteremia among individuals with and without end-stage renal disease: a Danish, population-based cohort study. BMC Infect Dis 2015;15:6.

12. Uchida D, Sasaki S, Kawarazaki $\mathrm{H}$, et al. Systemic inflammatory response syndrome is not an indicator of bacteremia in hemodialysis patients with native accesses: a multicenter study. Asaio $J$ 2017;63:501-6.
13. Sasaki S, Hasegawa T, Kawarazaki H, et al. Development and validation of a clinical prediction rule for bacteremia among maintenance hemodialysis patients in outpatient settings. PLoS One 2017; 12:e0169975.

14. Freund Y, Lemachatti N, Krastinova E, et al. Prognostic accuracy of sepsis-3 criteria for in-hospital mortality among patients with suspected infection presenting to the emergency department. JAMA 2017;317:301-8.

15. Williams JM, Greenslade JH, McKenzie JV, et al. Systemic inflammatory response syndrome, quick sequential organ function assessment, and organ dysfunction: insights from a prospective database of ed patients with infection. Chest 2017;151:586-96.

16. Wang JY, Chen $Y X$, Guo SB, et al. Predictive performance of quick Sepsis-related Organ Failure Assessment for mortality and ICU admission in patients with infection at the ED. Am J Emerg Med 2016;34:1788-93.

17. Churpek MM, Snyder A, Han X, et al. Quick Sepsis-related Organ Failure Assessment, Systemic Inflammatory Response Syndrome, and Early Warning Scores for Detecting Clinical Deterioration in Infected Patients outside the Intensive Care Unit. Am J Respir Crit Care Med 2017;195:906-11.

18. April MD, Aguirre J, Tannenbaum LI, et al. Sepsis Clinical Criteria in Emergency Department Patients Admitted to an Intensive Care Unit: An External Validation Study of Quick Sequential Organ Failure Assessment. J Emerg Med 2017;52:622-31.

19. Dorsett M, Kroll M, Smith CS, et al. qSOFA has poor sensitivity for prehospital identification of severe sepsis and septic shock. Prehosp Emerg Care 2017;21:489-97.

20. Singer AJ, Ng J, Thode HC, et al. Quick SOFA scores predict mortality in adult emergency department patients with and without suspected infection. Ann Emerg Med 2017;69:475-9.

21. Hwang SY, Jo IJ, Lee SU, et al. Low accuracy of positive qsofa criteria for predicting 28-day mortality in critically ill septic patients during the early period after emergency department presentation. Ann Emerg Med 2018;71:1-9.

22. Moons KG, Altman DG, Reitsma JB, et al. Transparent Reporting of a multivariable prediction model for Individual Prognosis or Diagnosis (TRIPOD): explanation and elaboration. Ann Intern Med 2015;162:W1-73.

23. Ohta T, Waga S, Handa W, et al. [New grading of level of disordered consiousness (author's transl)]. No Shinkei Geka 1974;2:623-7.

24. Shigematsu K, Nakano H, Watanabe $\mathrm{Y}$. The eye response test alone is sufficient to predict stroke outcome--reintroduction of Japan Coma Scale: a cohort study. BMJ Open 2013;3:e002736.

25. Hauser AB, Stinghen AE, Kato $\mathrm{S}$, et al. Characteristics and causes of immune dysfunction related to uremia and dialysis. Perit Dial Int 2008;28:S183-7.

26. Agarwal R, Nissenson AR, Batlle D, et al. Prevalence, treatment, and control of hypertension in chronic hemodialysis patients in the United States. Am J Med 2003:115:291-7.

27. Yamamoto S, Yamazaki S, Shimizu T, et al. Body temperature at the emergency department as a predictor of mortality in patients with bacterial infection. Medicine 2016;95:e3628. 